\title{
The frequency of grammatical classes in the production of verbal strings under two response sets
}

MARTIN ARLINSKY AND WILLIAM EPSTEIN

UNIVERSITY OF KANSAS

\begin{abstract}
Six groups, each consisting of $10 \mathrm{Ss}$, produced strings of 11,22 , or 33 words, under one of two sets of instructions: "structured" instructions which asked $S$ to produce a grammatically acceptable string; "unstructured instructions" which solicited a random, unconstrained string. Each word in S's production was classified into one of six grammatical classes. For the structured instructions the rank ordering of frequency of the six classes was functors (40\%), nouns $(25 \%)$, verbs $(13 \%)$, adjectives $(12 \%)$, pronouns $(6 \%)$, adverbs (3\%). For the unstructured instructions the rank ordering of frequency was nouns (68\%), adjectives $(12 \%)$, verbs $(11 \%)$, functors $(4 \%)$, adverbs $(1 \%)$, pronouns $(0.55 \%)$. The findings were discussed in relation to three questions.
\end{abstract}

\section{Introduetion}

This experiment was designed to determine the frequency of appearance of words, belonging to the various grammatical classes, in the verbal strings, which are produced by Ss under two sets of instructions. The significance of these data will be considered under three headings: (1) Evaluating the effects of syntactical structure on learning; (2) assessing the generality of the spew hypothesis as an account of certain characteristics of free verbal responding; and (3) specifying the distinguishing characteristics of grammatical utterances.

\section{Method}

General Plan. The experiment was a 2 by 3 factorial design. Two types of instructions and three list lengths were combined factorially.

Subjects. The Ss were students, whose native language was English. None of the students had prior laboratory experience with the procedures of this experiment. The Ss were tested in six groups, each containing 10 students.

Procedure. Sheets of ruled writing paper were distributed, and one of the following six versions of the instructions was read: unstructured instructions-"...write $(11,22,33)$ English words. They should be written as a random string, just as they come to mind, Do not use proper names..." structured instructions - "...write a grammatically correct English sentence of $(11,22,33)$ words. Construct the sentence with whatever words come to mind, but do not use proper names. Use commas sparingly, but do not use semicolons, colons, dashes, or other punctuation marks..." Ample time was allowed for completion of the task.

Scoring. Each word in the written protocols was classified in one of the following categories: noun, verb, adjective, adverb, pronoun and functor. The class of functors included articles, prepositions, conjunctions, interjections, quantity words and auxiliary verbs. This classification is based on Fries' (1952) analysis, but it also incorporates the modification introduced by Aborn et al (1959), regarding the combination of Fries' function words into one class. Two measures were derived from each protocol: the number of words in each grammatical class, and the percent of the total words in each class.
TABLE I

Mean Frequency of Occurrence of Words in Six Grammatical Classes

\begin{tabular}{lcccccc}
$\begin{array}{l}\text { Grammatical } \\
\text { Class }\end{array}$ & \multicolumn{2}{c}{$\begin{array}{c}\text { 11-Word String } \\
\text { Instructions } \\
\text { STR }\end{array}$} & UNSTR & \multicolumn{2}{c}{$\begin{array}{c}\text { 22-Word String } \\
\text { Instructions }\end{array}$} & \multicolumn{2}{c}{$\begin{array}{c}\text { 33-Word String } \\
\text { Instructions }\end{array}$} \\
\hline STnctors & 4.3 & 0.9 & 8.7 & 0.7 & 14.2 & 1.7 \\
Nouns & 2.8 & 8.2 & 5.1 & 16.7 & 8.8 & 18.7 \\
Verbs & 1.5 & 0.9 & 3.1 & 2.1 & 3.7 & 5.2 \\
Adjectives & 1.5 & 1.0 & 2.9 & 2.2 & 3.1 & 5.9 \\
Adverbs & 0.4 & 0.0 & 0.8 & 0.2 & 0.8 & 1.1 \\
Pronouns & 0.5 & 0.0 & 1.4 & 0.1 & 2.4 & 0.4 \\
\hline
\end{tabular}

\section{Results}

The mean number of words in each grammatical class is shown in Table 1. In Fig. 1 the mean for each class is expressed as a proportion of the total words, and is plotted against string length for the two instructional conditions.
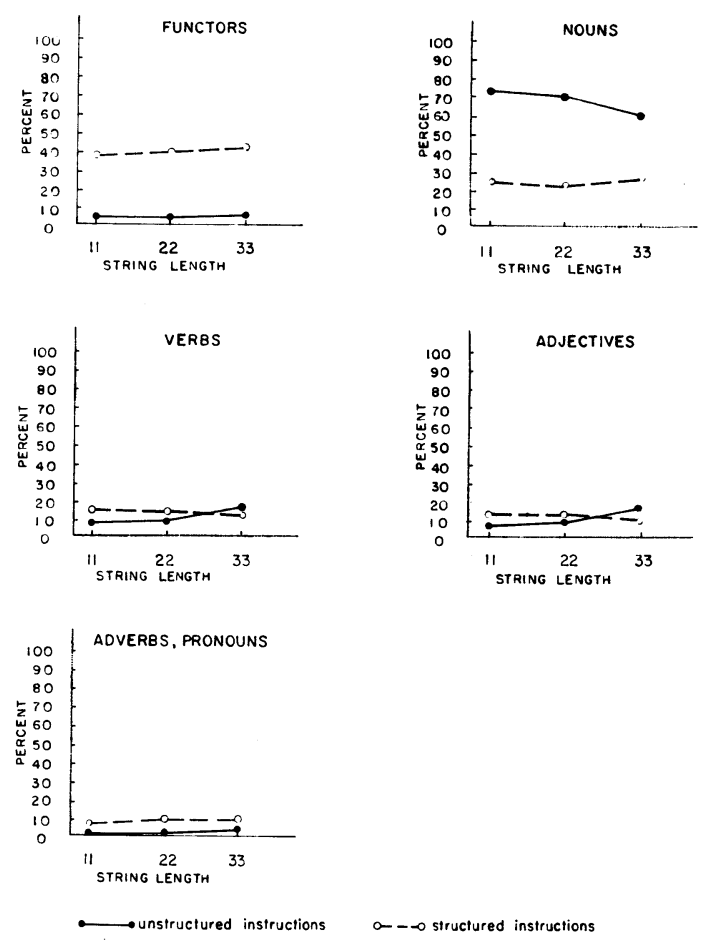

Fig. 1. The frequency of words in each of the grammatical classes expressed as a percent of the total words for each string length. 
(1) Functors. Table 1 shows that when "structured" instructions were given, the mean number of functors ranged from 4.3 to 14.4 , increasing in approximately linear fashion as a function of string length. In contrast, the means for the "unstructured" instructions ranged only from 0.8 to 1.2 , varying little as a function of string length. An analysis of variance showed that the main effects of instructions ( $F=639.04, \mathrm{df}=1 / 54, \mathrm{p}<.001)$ and string length ( $F=86.15, \mathrm{df}=2 / 54, \mathrm{p}<.001$ ), as well as the interaction between instructions and length $(\mathrm{F}=72.17, \mathrm{df}=2 / 54, \mathrm{p}<.001)$, were highly significant. The differences between the means for the three string lengths is significantly greater for the structured instructions than for the unstructured instructions.

(2) Nouns. Table 1 shows an overall tendency for the number of nouns to increase as a function of string length. For the structured instructions the means ranged from 2.8 to 8.8 . The means for the unstructured instructions were considerably higher, ranging from $\mathbf{8 . 2}$ to 18.7. An analysis of variance showed both main effects to be highly significant (Instructions, $F=136.65$, $\mathrm{df}=1 / 54, \mathrm{p}<.001$; string length, $F=39.04, \mathrm{df}=2 / 54$, $\mathrm{p}<.001)$. The interaction effect was also significant ( $\mathrm{F}=5.70, \mathrm{df}=2 / 54, \mathrm{p}<.01)$.

(3) Verbs, adjectives, adverbs, pronouns. Inspection of Table 1 shows that verbs and adjectives occurred with comparable frequency. Verbs accounted for $12.07 \%$, and adjectives for $12.20 \%$ of the total words. Analyses of variance showed a significant trend for the number of verbs and adjectives to increase with increasing string length ( $F$ verbs $=16.02, d f=2 / 54, p<.001 ; F$ adjectives $=14.07, \mathrm{df}=2 / 54, \mathrm{p}<.001)$. The differences between instructional conditions were not significant. Adverbs and pronouns were very infrequent and their frequency of occurrence did not vary as a function of instructions or string length.

The curves presented in Fig. 1 show that the proportion of words in each grammatical class remained approximately constant over the variations of string length. This trend is especially evident in the structured productions, but it is also obvious in the curves for functor and noun frequency in the unstructured productions.

\section{Diseussion}

The results show that the frequency of occurrence of words in the various grammatical classes is not the same for the two instruetional conditions. The chief difference concerns the frequency of functors and nouns. Functors constituted $40 \%$ of the words produced under "structured" instructions, but only $4 \%$ of the words produced under "unstructured" instructions. As for nouns, the relative frequency was reversed. In the structured productions nouns made up $25 \%$ of the words, while in the unstructured productions $68 \%$ of the words were nouns. The differences for the remaining four form clisses were of lesser magnitude, and inconsistent over string length.

These results have implications for a number of questions. Three of these implications will be considered here: (1) Asscssing the influcnic of syntactical struiture on learning. Evidence has been presented that syntactical strings are easier to learn than nonsyntiletical strings composed of the same words (Epstein, 1961, 1962; Marks \& Miller, 196t). An important part of the evidence has consisted of showing that, after a given number of learning trials, more items are correctly reproduced from a syntactical string than a nonsyntactical string. However, the present results imply that the difference in the number of correctly produced items may not be due ixclusively to differences in the amount of specific learning of the test material. The results suggest that if the $S$, who learns the syntactical material, recognizes that the series is syntactical, then he will tend to distribute the words he produces between the various form classes differently than a $S$ who does not discern any syntactical constraints. For example, the $S$ who considers his task to be the reproduction of a grammatical series will tend to produce functors during the recall test. Since ne number of different functors is relutively small this response tendency may produce an enhancement of correct functor production. No such tendency will exist in the S who learns the nonsyntactical material. On the other hand, the $S$ who is given unstructured material to learn may be inclined to produce large numbers of nouns. This tendency will hinder correct reproduction since it will favor the production of nouns in excess of their "itual frequency. The net results of these response tendencies would be to inflate the difference between the test scores, and produce an exagerated impression of the influence of syntactical structure on the acquisition of the specific test material.

(2) The generality of the spew hypothesis: The spew hypothesis (Underwood \& Schulz, 1960) states that the frequency and order of emission of verbal items, in free responding, will be positively correluted with the frequency of occurrence of the item in the linguage. On this hypothesis, one would expect that functors, which are the most frequently encountered words in the English language, would occur very frequently in the productions of the Ss who received "unstructured" instructions. Obviously, this expectation was not borne out, since functors constituted only $4 \%$ of the words produced by our Ss. ${ }^{1}$ Apparently it is not true that the most frequently occurring words in the language are also the words which occur most frequently in S's unrestricted verbal productions. It should be noted, however, that functors may constitute a special case, since functors are not usually emitted as individual words out of sentential context.

(3) The distinguishing characteristics of grammatical utterances: In the present study, the only significant differences between the grammatical and ungrammatical productions involved the frequency of occurrence of functors and nouns. The frequencies obtained in our experiment are in agreement with the frequencies reported by French et al (1930) and Fries (1952) based on random sampling of words in telephone conversations, and Aborn et al (1959) analysis of contextual constraint. The differences in functor frequency implicuates the crucial role of functors. ${ }^{2}$ These words play a unique role in specifying the logical and temporal relations which constitute a grammatical utterance. The presence of functors in appropriate number may be one of the distinctive features of grammatical as opposed to ungrammatical utterances.

\section{Referenees}

Aborn, M., Rubenstein, J., \& Sterling, T. Sources of contextual constraint upon words in sentences. J. exp. Psychol., 1959, 57, 171-180.

Epstein, W. The influence of syntactical structure on learning. Amer. J. Psychol., 1961, 74, 80-85.

Epstein, W. A further study of the influence of syntactical structure on learning. Amer. J. Psychol., 1962, 75, 121-126.

French, N. R., Carter, C. W., \& Koenig, W. The words and sounds of telephone conversations. Bell Sys. tech. J., 1930, 9, 290-324.

Fries, C. C. The structure of English. New York: Harcourt, Brace, 1952.

Marks, L. E., \& Miller, G. A. The role of semantic and syntactic constraints in the memorization of English sentences. J. verbal Learn. verbal behav., 1964, 3, 1-5.

Underwood, B. J., \& Schulz, R. W. Meaningfulness and verbal learning. Chicago: Lippincott, 1960.

\section{Notes}

1. The protocols produced by the three "unstructured" groups were examined to determine whether the functors which were produced appeared early in the strings. Forty-five percent of the functors appeared in the first third of the strings, $25 \%$ in the second third, and $30 \%$ in the last third.

2. The high incidence of nouns in the unstructured series is of less significance for the question of grammaticality. 